Check for updates

Cite this: RSC Adv., 2018, 8, 17629

\title{
Preparation of boron-doped mesoporous carbon with aromatic compounds as expanding agents
}

\begin{abstract}
Yan Zhang, (D)* Bing'e Ma and Yujian Liu
Boron-doped ordered mesoporous carbon (B-OMC) was synthesized using the aromatic compounds benzene, 1,3,5-trimethylbenzene, 1,3,5-triethylbenzene and 1,3,5-triisopropylbenzene as expanding agents. The expanding mechanism as well as the effect of the expanding agent molecule on the properties of B-OMCs were studied. Compared with the unmodified one, the order of B-OMCs treated with aromatic compounds is improved significantly. In addition, along with the increase in hydrophobicity and steric hindrance of the expanding agents, the pore size and pore volume of B-OMCs increase, while their surface area and specific capacitance increase first, and then drop off slightly. The obtained B-OMC-TEB has a high boron content (1.54 wt\%), the largest surface area $\left(693 \mathrm{~m}^{2} \mathrm{~g}^{-1}\right)$, a much better electrochemical performance and the highest specific capacitance $\left(290 \mathrm{~F} \mathrm{~g}^{-1}\right), 30 \%$ higher than that of ordinary B-OMC. Furthermore, the specific capacitance can be maintained at $155 \mathrm{~F}$ $\mathrm{g}^{-1}$ even at a high current density of $20 \mathrm{~A} \mathrm{~g}^{-1}$, indicating that it has a high capacitance retention rate.
\end{abstract}

Received 15th February 2018 Accepted 3rd May 2018

DOI: 10.1039/c8ra01455h

rsc.li/rsc-advances

form hydrogen bonds with the template and the poor solubility of boron modified phenolic resin. ${ }^{21,22}$

The size of formed micelles determines the pore size of the final mesoporous material. ${ }^{23}$ Hydrophobic compounds can be dissolved in the hydrophobic core of surfactant micelles, causing the micelles to expand, thereby increasing the pore size of the mesoporous material. ${ }^{24}$ Some studies have attempted to incorporate and localize hydrophobic compounds into Pluronic/ surfactant mixed micelles. ${ }^{25}$ For example, by using cetyltrimethylammonium bromide (CTMABr) as the template and decane as the expanding agent, the pore size of MCM-41 expanded from $2.6 \mathrm{~nm}$ to more than $4.0 \mathrm{~nm}$, and its specific surface area increased from $432 \mathrm{~m}^{2} \mathrm{~g}^{-1}$ to $802 \mathrm{~m}^{2} \mathrm{~g}^{-1}$. ${ }^{26}$ In addition, aromatic compounds also serve as additives to enlarge the pore size of mesoporous silicon and mesoporous hydroxyapatite. ${ }^{27-29}$

Based on our previous research, aromatic compounds with rigid, stable benzene rings and hydrophobic hydrocarbon substituents, benzene, 1,3,5-trimethylbenzene, 1,3,5-triethylbenzene and 1,3,5-triisopropylbenzene, were chosen as expanding agents to further increase the pore size and the specific surface area of B-OMCs. The influence of aromatic compounds on the mesoporous structure and characteristics of B-OMCs and the expanding mechanism were investigated. Additionally, the electrochemical properties of the obtained BOMCs were evaluated.

\section{Experimental}

\subsection{Materials}

Tri-block polymer F108 $\left(\mathrm{PEO}_{132} \mathrm{PPO}_{50} \mathrm{PEO}_{132}\right)$ was provided by Sigma-Aldrich with the average molecular weight 14600. 
Formaldehyde (37 wt\%), phenol, hydrochloric acid solution, boric acid and benzene (Be) were purchased from Ling Feng Chemical Reagent Co., Ltd. Aromatic compounds 1,3,5-trimethylbenzene (TMB), 1,3,5-triethylbenzene (TEB) and 1,3,5-triisopropylbenzene (TiPB) were obtained from Admas Reagent Co., Ltd.

\subsection{Preparation of B-OMCs}

Boron-doped ordered mesoporous carbon materials were prepared according to the procedure that we used before. ${ }^{30}$ Boron phenolic resin (BPF) was chosen as the precursor, F108 as the soft template, and aromatic compounds as the expanding agent. At first, F108 was dissolved in ethanol with the aromatic expanding agent together. Then the BPF solution was added into the mixture with $\mathrm{HCl}$ as the self-assembly regulator, and the $\mathrm{pH}$ of the system was about 4 . After evaporation at $25^{\circ} \mathrm{C}$ for $24 \mathrm{~h}$, and cross-linking at $100{ }^{\circ} \mathrm{C}$ for $24 \mathrm{~h}$, the complex was calcined at $800{ }^{\circ} \mathrm{C}$ for $2 \mathrm{~h}$ with a heating rate of $1{ }^{\circ} \mathrm{C} \mathrm{min} \min ^{-1}$ in a $\mathrm{N}_{2}$ atmosphere. The samples were named B-OMC- $x$, with $x$ representing the expanding agent.

\subsection{Characterization}

Small angle X-ray powder diffraction (SXRD) patterns were collected with a Rigaku D/max-2550 diffractometer (Japan) using $\mathrm{Cu}-\mathrm{K} \alpha$ as the radiation $(\lambda=0.154 \mathrm{~nm})$. Transmission electron microscopy images were observed using a JEM-1400 microscope (Japan) at $100 \mathrm{kV}$. Nitrogen adsorption/ desorption measurements were achieved on an ASAP 2020 (US) at $77 \mathrm{~K}$. The sample was degassed under vacuum at $200{ }^{\circ} \mathrm{C}$ for $6 \mathrm{~h}$ prior to the test. The pore size distribution of the sample was calculated from the adsorption branches of the isotherms according to the BJH method, and the BET specific surface area was obtained by the BET method. XPS measurements were conducted using a Thermo ESCALAB 250 (US) at $150 \mathrm{~W}$.

\subsection{Electrochemical measurements}

Working electrodes were prepared according to the standard method. ${ }^{31}$ A mixture of active materials ( $80 \mathrm{wt} \%$ ), acetylene black (15 wt\%) and polytetrafluoroethylene (5 wt\%) was homogenized in a mortar, then punched into pellets at a pressure of $14 \mathrm{MPa}$. Each working electrode was dried for $12 \mathrm{~h}$ at $110{ }^{\circ} \mathrm{C}$, then impregnated with an electrolyte for testing. The test was conducted in a three-electrode system in $1 \mathrm{M} \mathrm{H}_{2} \mathrm{SO}_{4}$ solution. A platinum electrode was taken as the counter electrode and a saturated calomel electrode as the reference electrode.

Cyclic voltammograms (CV) and galvanostatic chargedischarge (GCD) measurements were recorded on an electrochemical workstation (CHI660D, China) in a potential window of 0-0.9 V. The specific capacitance of mesoporous carbon was obtained from the $C_{\mathrm{m}}=I \Delta t /(m \Delta U)$ equation, where $C_{\mathrm{m}}$ is the specific capacitance, $I$ represents the discharge current in an electrochemical system, $\Delta t$ is the discharge time, $m$ is the mass of mesoporous carbon on an electrode, and $\Delta U$ is the discharge voltage window.

\section{Results and discussion}

\subsection{Textural and structural characterization}

All of the samples have an obvious diffraction peak at around $2 \theta=1^{\circ}$, implying they have ordered mesoporous structures, as shown in Fig. 1. Compared with the unmodified B-OMC, the diffraction peaks of the mesoporous carbon materials with an expanding agent became much stronger and sharper, indicating that they have a better ordered mesostructure. This can be attributed to the high thermal stability of the benzene rings of the expanding agents, which tend to prevent the mesoporous carbon channels from collapsing during the calcination process.

With the number of hydrocarbon substituent groups increasing, the hydrophobicity as well as the molecular size of the aromatic expanding agents increases in the order of benzene $<$ TMB $<$ TEB $<$ TiPB. From B-OMC-Be to B-OMC-TiPB, the (100) peak of the B-OMCs shifts to a lower angle (from $0.902^{\circ}$ to $0.794^{\circ}$ ), suggesting that the interplanar spacing $d$ of the samples has been augmented. It also demonstrates that contraction of mesoporous carbon channels can be reduced by introducing aromatic expanding agents and the shrinkage decreases with an increase in the molecular size of the expanding agents. Among them, B-OMC-TiPB has the lowest shrinkage.

The morphologies of the as-prepared B-OMCs are displayed in Fig. 2. All of the samples possess $2 \mathrm{D}$ hexagonal $\mathrm{p} 6 \mathrm{~mm}$ mesostructures. Nevertheless, the order of B-OMC without an expanding agent is inferior to that of the modified B-OMCs, which can be ascribed to a shortage of rigid aromatic rings that restrict the channel from collapsing during calcination. These results are in line with those of XRD.

The chemical composition of B-OMC-TEB was further analysed by XPS and the results are shown in Fig. 3 and Table 1. Boron $(192 \mathrm{eV})$ and oxygen $(533 \mathrm{eV})$ were introduced into the carbon framework successfully. In addition, the B 1s spectrum can be fitted by two peaks at $191.98 \mathrm{eV}$ and $192.67 \mathrm{eV}$, corresponding to $-\mathrm{O}-\mathrm{B}-\mathrm{O}-\mathrm{C}^{9}$ and $\mathrm{B}_{2} \mathrm{O}_{3}$. The high content of $\mathrm{B}_{2} \mathrm{O}_{3}$ should be derived from the partial decomposition of triphenyl borate $^{32}$ and that of unreacted boric acid during calcination. ${ }^{22}$

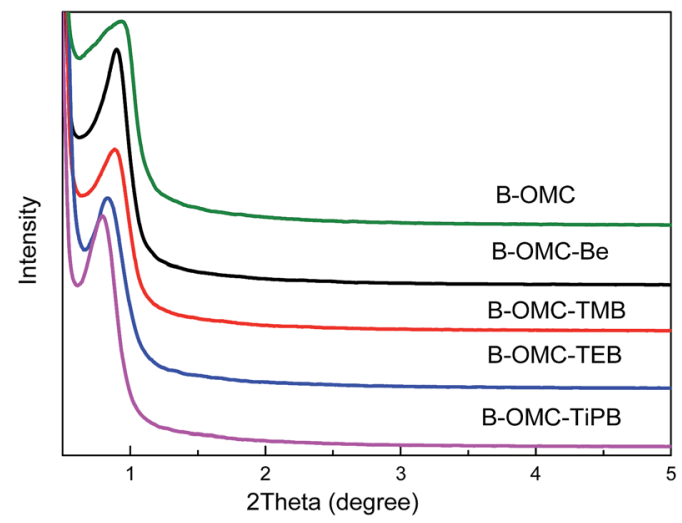

Fig. 1 Small-angel XRD patterns of the B-OMCs with different expanding agents, calcined at $800^{\circ} \mathrm{C}$ under $\mathrm{N}_{2}$. 


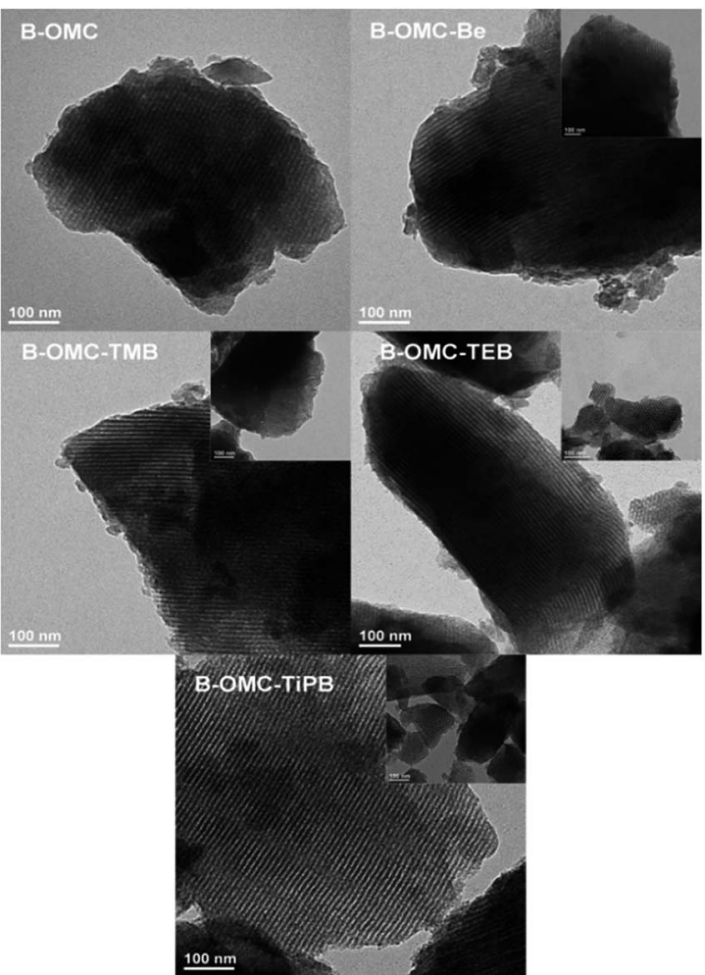

Fig. 2 TEM images of the B-OMCs with different expanding agents, calcined at $800^{\circ} \mathrm{C}$ under $\mathrm{N}_{2}$

Meanwhile, $\mathrm{B}_{2} \mathrm{O}_{3}$ with good fluidity prefers to migrate to the surface of mesoporous carbon. The $\mathrm{O} 1 \mathrm{~s}$ spectrum is deconvoluted into four peaks located at $531.43 \mathrm{eV}(\mathrm{C}=\mathrm{O}), 532.60 \mathrm{eV}(\mathrm{C}-$ $\mathrm{O}), 533.60 \mathrm{eV}(\mathrm{C}-\mathrm{OH})$ and $536.20\left(\mathrm{H}_{2} \mathrm{O}\right) .{ }^{33}$ The amounts of boron and oxygen for B-OMC-TEB were $1.54 \mathrm{wt} \%$ and $16.33 \mathrm{wt} \%$, which would contribute to the introduction of a pseudocapacitance effect, ${ }^{12}$ and obtaining a high capacitance retention at a high scan rate. ${ }^{34}$

As presented in Fig. 4, the $\mathrm{N}_{2}$ adsorption/desorption isotherms of all of the samples are of type IV and have welldefined H1 hysteresis loops, signifying that mesoporous characters have been obtained. Moreover, their pore size distributions are narrow. The structural parameters of the B-OMCs are listed in Table 2. The pore size and BET surface area of B-OMC$\mathrm{Be}$ are very close to those of B-OMC. For B-OMC-TMB, B-OMCTEB and B-OMC-TiPB, however, their BET surface area increased from $603 \mathrm{~m}^{2} \mathrm{~g}^{-1}$ to $693 \mathrm{~m}^{2} \mathrm{~g}^{-1}$, and their pore size increased from $4 \mathrm{~nm}$ to $4.9 \mathrm{~nm}$ with an increasing number of hydrocarbons in the substituent groups. Compared with that of unmodified B-OMC, the surface area of B-OMC-TEB was raised by $15 \%$, and the pore size of B-OMC-TiPB increased by $22 \%$.

\subsection{The expansion mechanism of the aromatic compounds}

One of the important conditions for the preparation of mesoporous carbon via a self-assembly method is that stable micelles can be formed in the solvent. ${ }^{35,36}$ The pore size of mesoporous carbon mainly depends on the size of the micelle. The BET specific surface areas and pore sizes of most of the B-OMC- $x$
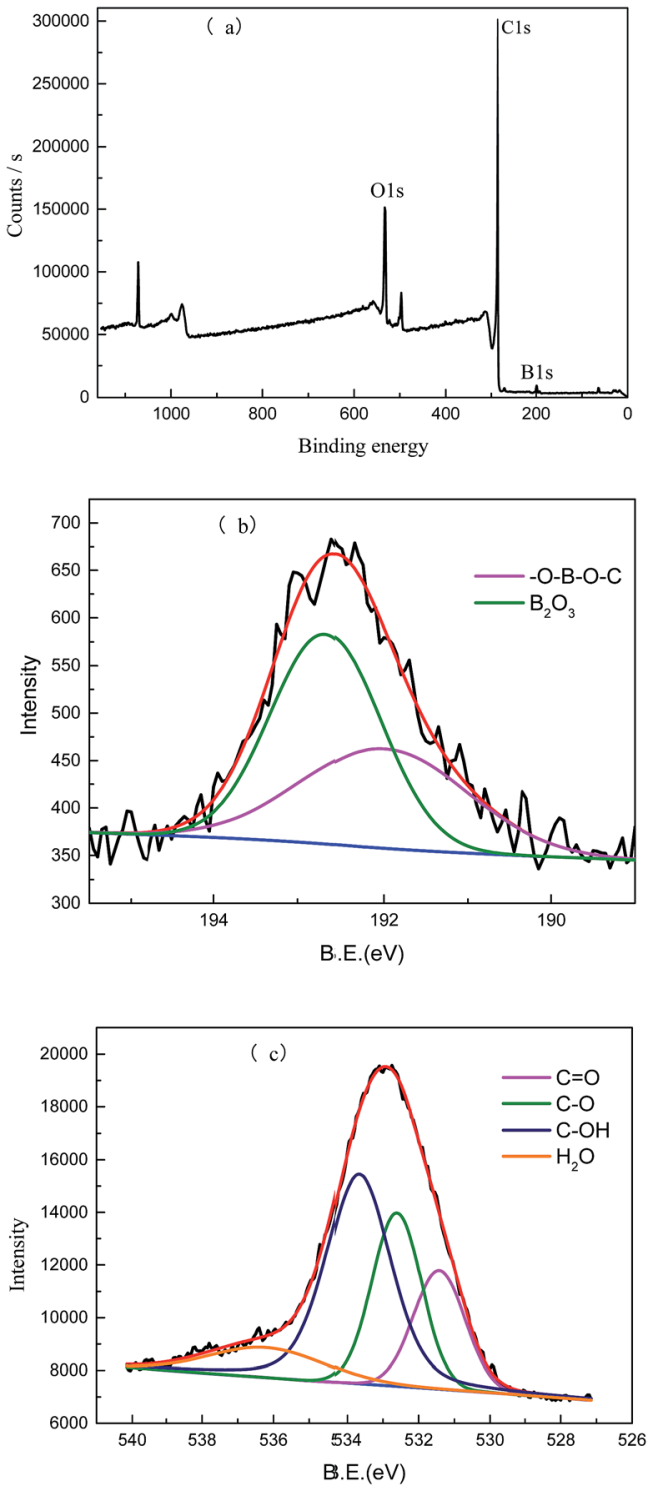

Fig. 3 XPS spectra of B-OMCs (a), B 1s (b) and O 1s (c).

Table 1 XPS results of B-OMC

\begin{tabular}{lll}
\hline Element & B.E. $(\mathrm{eV})$ & Content $(\mathrm{wt} \%)$ \\
\hline B & $191.98(-\mathrm{O}-\mathrm{B}-\mathrm{O}-\mathrm{C})$ & 0.66 \\
& $192.67\left(\mathrm{~B}_{2} \mathrm{O}_{3}\right)$ & 0.88 \\
Total content & - & 1.54 \\
O & $531.43(\mathrm{C}=\mathrm{O})$ & 3.00 \\
& $532.60(\mathrm{C}-\mathrm{O})$ & 4.21 \\
& $533.60(\mathrm{C}-\mathrm{OH})$ & 7.45 \\
Total content & $536.20\left(\mathrm{H}_{2} \mathrm{O}\right)$ & 1.67 \\
& - & 16.33
\end{tabular}

samples are larger than that of B-OMC. The structures of additive molecules would influence the surfactant template curvatures in liquid crystals. ${ }^{37}$ Hydrophilic additive molecules such as dodecanol are usually located in the outer hydrophilic chain gaps, exhibiting a "penetration effect", while hydrophobic 

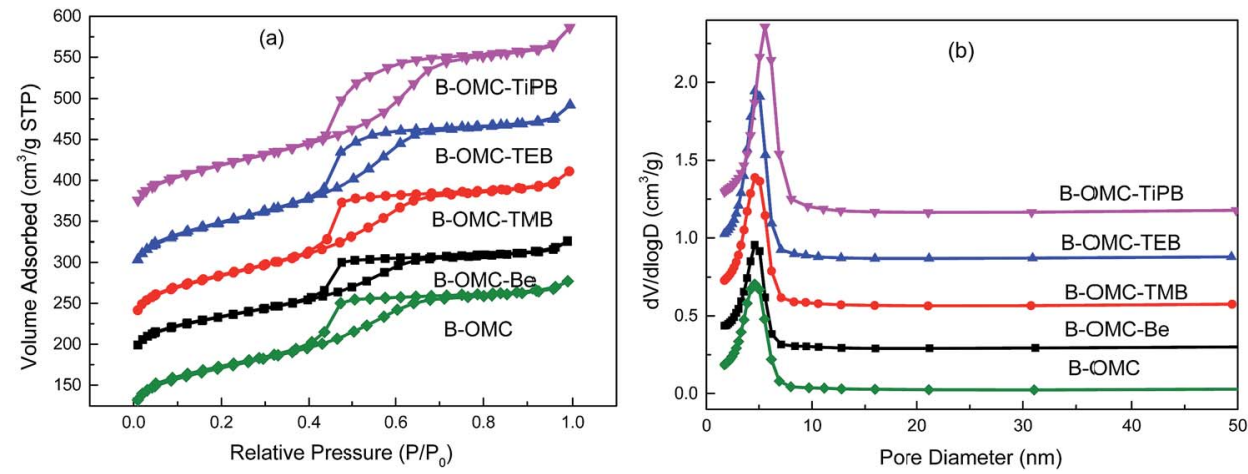

Fig. 4 Adsorption-desorption isotherms (a) and pore size distributions (b) of the B-OMCs with different expanding agents, calcined at $800{ }^{\circ} \mathrm{C}$ under $\mathrm{N}_{2}$.

Table 2 The structural properties and specific capacitances of the B-OMCs

\begin{tabular}{|c|c|c|c|c|c|c|c|c|}
\hline Sample & $S_{\mathrm{BET}}{ }^{a}\left(\mathrm{~m}^{2} \mathrm{~g}^{-1}\right)$ & $S_{\text {mic }}\left(\mathrm{m}^{2} \mathrm{~g}^{-1}\right)$ & $V_{\mathrm{p}}^{b}\left(\mathrm{~m}^{3} \mathrm{~g}^{-1}\right)$ & $V_{\text {mic }}\left(\mathrm{m}^{3} \mathrm{~g}^{-1}\right)$ & $D_{\mathrm{p}}^{b}(\mathrm{~nm})$ & $\begin{array}{l}\text { Current density } \\
\left(\mathrm{A} \mathrm{g}^{-1}\right)\end{array}$ & $C^{c}\left(\mathrm{~F} \mathrm{~g}^{-1}\right)$ & $d^{d}$ \\
\hline B-OMC & 603 & 315 & 0.44 & 0.17 & 4.0 & 0.86 & 222 & 9.39 \\
\hline B-OMC-TMB & 666 & 351 & 0.50 & 0.19 & 4.2 & 0.90 & 235 & 9.98 \\
\hline B-OMC-TEB & 693 & 356 & 0.55 & 0.19 & 4.3 & 1.09 & 290 & 10.66 \\
\hline B-OMC-TiPB & 682 & 361 & 0.55 & 0.12 & 4.9 & 1.00 & 261 & 1.11 \\
\hline
\end{tabular}

${ }^{a}$ The BET surface areas of the samples were calculated by using the BET method. ${ }^{b}$ The pore size distributions were derived from the BJH method.

${ }^{c}$ The specific capacitance per gram measured at the current density of $0.2 \mathrm{~A} \mathrm{~g}^{-1}$. ${ }^{d}$ Calculated according to the Bragg's law $2 d \sin \theta=n \lambda$.

compounds like saturated hydrocarbons tend to enter the core of the micelle, showing a "swelling effect".

A possible expansion mechanism of aromatic compounds has been derived, as shown in Scheme 1. At first, the micelles of template $\mathrm{F} 108$ are formed in ethanol solution with a core radius $(r)$. After adding benzene into the F108 micelle solution, the rigid and bulky benzene rings restrict it from entering the core of the micelle, and it just stays in the palisade layer. Therefore, the core radius of the micelles remains the same. However, the strong electrostatic repulsion and volume effect of benzene helps to increase the surface area of aggregates between the PEO chains. So, the $S_{\text {mic }}$ and $V_{\text {mic }}$ of B-OMC-Be are augmented. With an increasing number of hydrophobic hydrocarbons in the substituent groups, expanding agent molecules can not only be located at the palisade layer, but can also be dragged into the core of the micelles. The core radius of B-OMC- $x$ was raised from $r$ to $R$, and the pore size of the samples increased with the rising hydrophobic and steric hindrance of the aromatic compounds. The expanding agents TMB, TEB and TiPB exhibit both penetration effects and swelling effects.

\subsection{Electrochemical properties}

The electrochemical properties of the samples modified by expanding agents were investigated for use as electrode materials for supercapacitors. Fig. 5 exhibits the $\mathrm{CV}$ curves of the BOMCs at a scan rate of $5 \mathrm{mV} \mathrm{s}^{-1}$ in $1 \mathrm{M} \mathrm{H}_{2} \mathrm{SO}_{4}$, and the GCD measurements at a current density of $0.2 \mathrm{~A} \mathrm{~g}^{-1}$.

The symmetrical and rectangular CV curves of all of the samples in Fig. 5(a) imply ideal double layer capacitive

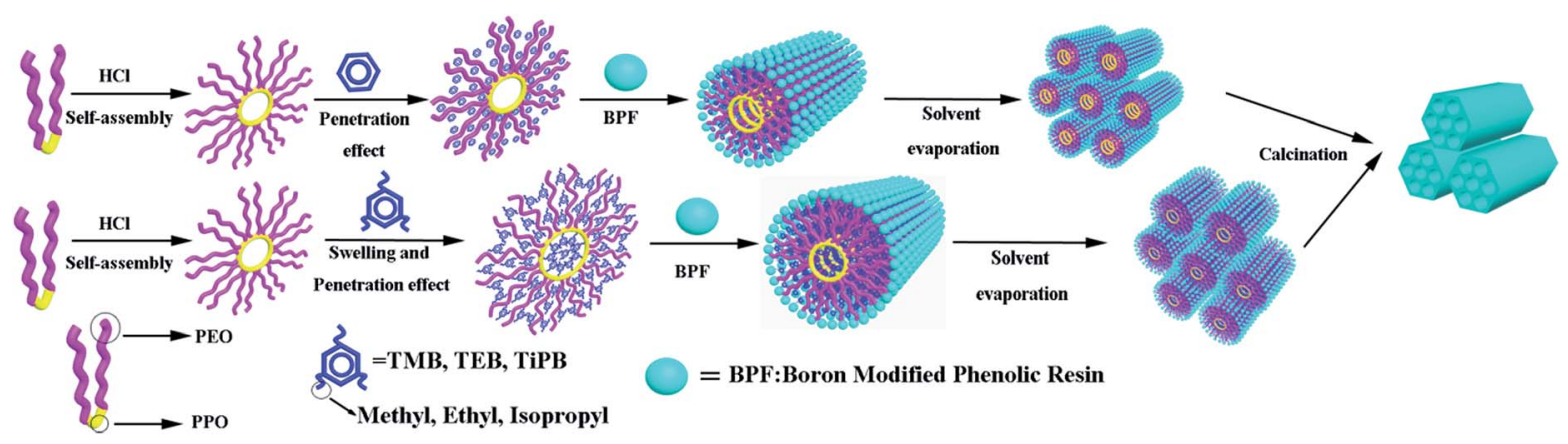

Scheme 1 The behavior of aromatic expanding agents in the micelle during EISA. 

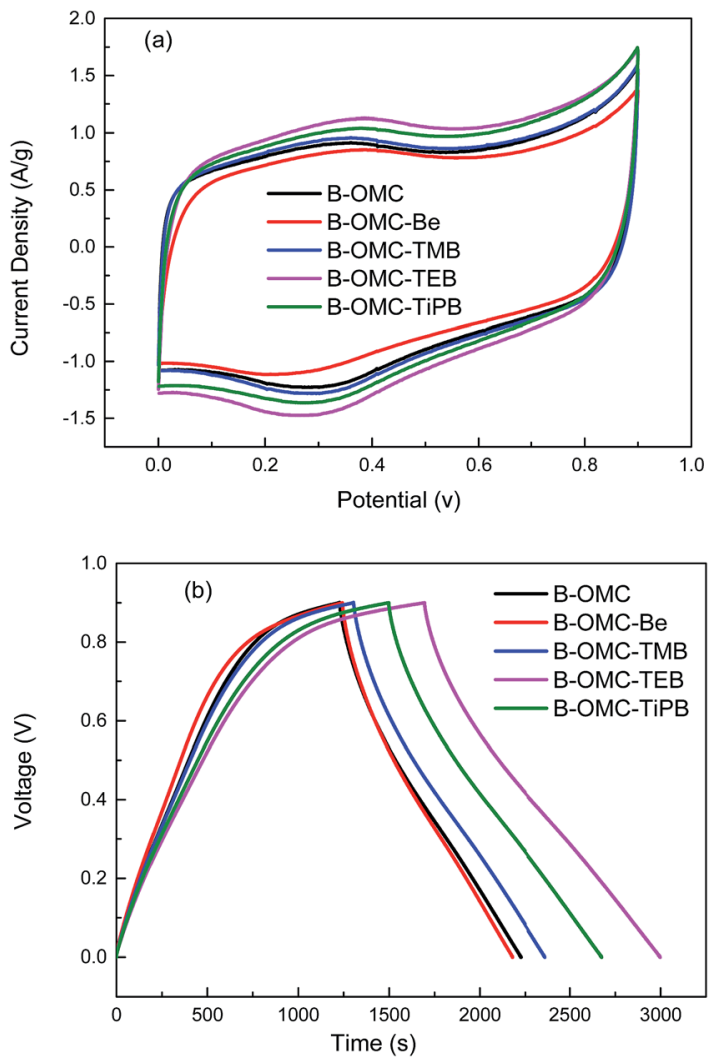

Fig. $5 \mathrm{CV}$ curves with a scan rate of $5 \mathrm{mV} \mathrm{s}^{-1}$ (a), and GCD curves at a current density of $0.2 \mathrm{~A} \mathrm{~g}^{-1}$ (b) of the B-OMCs with different expanding agents, calcined at $800{ }^{\circ} \mathrm{C}$ under $\mathrm{N}_{2}$, recorded in $1 \mathrm{M}$ $\mathrm{H}_{2} \mathrm{SO}_{4}$.

properties. Furthermore, with increasing hydrophobic and steric hindrance of the expanding agents, the current density of the samples increases gradually, then decreases slightly. BOMC-TEB possesses the maximum response current density of $1.09 \mathrm{~A} \mathrm{~g}^{-1}$. From Fig. 5(b), it is found that the B-OMCs with aromatic compounds as the expanding agent will result in the specific capacitance increasing from $222 \mathrm{~F} \mathrm{~g}^{-1}$ (B-OMC) to $290 \mathrm{~F}$ $\mathrm{g}^{-1}$ (B-OMC-TEB). This is also much higher than the previously reported data for B-OMCs $\left(128-175 \mathrm{~F} \mathrm{~g}^{-1}\right),{ }^{38-40}$ as well as our previous results $\left(200 \mathrm{~F} \mathrm{~g}^{-1}\right) .^{30}$ The large specific surface area, suitable pore size, and narrow pore size distribution of B-OMCTEB are beneficial for enhancing the electrochemical properties. Furthermore, improving the graphitization of B-OMCs would also lead to a high conductivity. ${ }^{41}$

The specific capacitance of mesoporous carbon at different current densities was also investigated, and the results are shown in Fig. 6. As the current density increased from $0.2 \mathrm{~A} \mathrm{~g}^{-1}$ to $20 \mathrm{~A} \mathrm{~g}^{-1}$, the specific capacitances of the B-OMCs reduced gradually, because the large current density would increase the resistance of the electrolyte ions in the channels. While the specific capacitance of B-OMC-TEB was $182 \mathrm{~F} \mathrm{~g}^{-1}$ at a high current density of $5 \mathrm{~A} \mathrm{~g}^{-1}$, this is higher than that of unmodified B-OMC $\left(126 \mathrm{~F} \mathrm{~g}^{-1}\right)$ and the previously reported data $\left(108 \mathrm{~F} \mathrm{~g}^{-1}\right.$, at $\left.5 \mathrm{~A} \mathrm{~g}^{-1}\right){ }^{31}$ Furthermore, its specific capacitance was maintained at $155 \mathrm{~F} \mathrm{~g}^{-1}$, even at a current

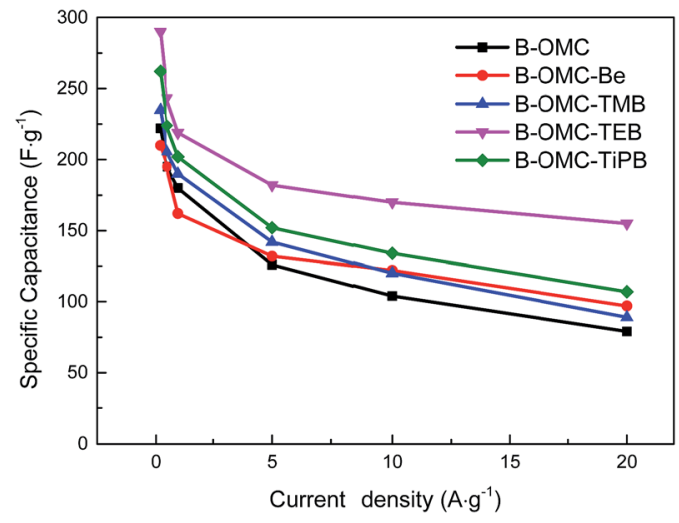

Fig. 6 The specific capacitance of the B-OMCs at different current densities.

density as high as $20 \mathrm{~A} \mathrm{~g}^{-1}$, indicating that it has a high capacitance retention rate.

\section{Conclusions}

Boron-doped mesoporous carbon materials with ideal mesoporosity were synthesized using aromatic compounds as expanding agents. TMB, TEB and TiPB exhibited both penetration effects and swelling effects. Along with the increase in hydrophobicity and steric hindrance of the expanding agents, the pore size and pore volume of the B-OMCs increased gradually, but the surface area increased first, and then dropped off slightly. B-OMC-TEB has the highest surface area $\left(693 \mathrm{~m}^{2} \mathrm{~g}^{-1}\right)$ and a well-ordered mesoporosity. Additionally, it also possesses the largest capacitance performance $\left(290 \mathrm{~F} \mathrm{~g}^{-1}\right), 30 \%$ higher than that of unmodified B-OMC, and $65 \%$ higher than the reported data. The specific capacitance can be maintained at 155 $\mathrm{F} \mathrm{g}^{-1}$ at a current density of $20 \mathrm{~A} \mathrm{~g}^{-1}$, indicating that it has a high capacitance retention rate.

\section{Conflicts of interest}

There are no existing conflicts of interest in the submission, and the manuscript has been approved for publication by all authors.

\section{Acknowledgements}

This work was supported by the National Natural Science Foundation of China (51303054) and Fundamental Research Funds for the Central Universities (222201817001).

\section{References}

1 M. E. Davis, Nature, 2002, 417, 813-821.

2 M. Enterría, M. F. R. Pereira, J. I. Martins and J. L. Figueiredo, Carbon, 2015, 95, 72-83.

3 A. Prabhu, A. A. Shoaibi and C. Srinivasakannan, Mater. Lett., 2014, 136, 81-84. 
4 S. E. Elaigwu, G. Kyriakou, T. J. Prior and G. M. Greenway, Mater. Lett., 2014, 123, 198-201.

5 S. H. Liu and J. R. Wu, Int. J. Hydrogen Energy, 2011, 36, 8793.

6 C. He and X. Hu, Ind. Eng. Chem. Res., 2011, 50, 14070-14083.

7 X. Zhang, R. F. Guan, D. Q. Wu and K. Y. Chan, J. Mol. Catal. B: Enzym., 2005, 33, 43-50.

8 A. Stein, B. J. Melde and R. C. Schroden, Adv. Mater., 2000, 12, 1403-1419.

9 D. W. Wang, F. Li, Z. G. Chen, G. Q. Lu and H. M. Cheng, Chem. Mater., 2008, 20, 7195-7200.

10 J. Gao, X. Y. Wang, Y. W. Zhang, J. Liu, Q. Lu and M. Liu, Electrochim. Acta, 2016, 207, 266-274.

11 W. Lu, M. Liu, L. Miao, D. Zhu, X. Wang, H. Duan, Z. Wang, L. Li, Z. Xu, L. Gan and L. Chen, Electrochim. Acta, 2016, 205, 132-141.

12 Y. Song, G. Liu and Z. Y. Yuan, RSC Adv., 2016, 6, 9463694642.

13 J. Zhang, N. Nie, Y. Liu, J. Wang, F. Yu, J. Gu and W. Li, ACS Appl. Mater. Interfaces, 2015, 7, 20134-20143.

14 H. Chen, Y. Xiong, T. Yu, P. Zhu, X. Yan, Z. Wang and S. Guan, Carbon, 2017, 113, 266-273.

15 J. Gao, X. Wang, Y. Zhang, J. Liu, Q. Lu and M. Liu, Electrochim. Acta, 2016, 207, 266-274.

16 K. Wang, H. Wang and Y. B. Cheng, J. Sol-Gel Sci. Technol., 2017, 82, 664-674.

17 Y. Maletin, P. Novak, E. Shembel, V. Izotov, N. Strizhakova, A. Mironova, V. Danilin and S. Podmogilny, Appl. Phys. A: Mater. Sci. Process., 2005, 82, 653-657.

18 J. Gamby, P. L. Taberna, P. Simon, J. F. Fauvarque and M. Chesneau, J. Power Sources, 2001, 101, 109-116.

19 D. Qu and S. Hang, J. Power Sources, 1998, 74, 99-107.

20 H. Shi, Electrochim. Acta, 1996, 41, 1633-1639.

21 J. Su, X. Cao, J. Wu, C. Jin, J. H. Tian and R. Yang, RSC Adv., 2016, 6, 24728-24737.

22 X. Zhai, Y. Song, J. Liu, P. Li, M. Zhong, C. Ma, H. Wang, Q. Guo and L. Zhi, J. Electrochem.Soc., 2012, 159, E177-E182. 23 A. Corma, Chem. Rev., 1997, 97, 2373-2420.
24 J. L. B. And and B. L. Su, Langmuir, 2002, 18, 5303-5308.

25 A. H. E. Müller and O. Borisov, Adv. Polym. Sci., 2011, 241, IXXI.

26 J. L. Blin, C. Otjacques, A. G. Herrier and B. L. Su, Langmuir, 2000, 16, 4229-4236.

27 J. Fan, C. Z. Yu, L. M. Wang, B. Tu, D. Y. Zhao, Y. Sakamoto and O. Terasaki, J. Am. Chem. Soc., 2001, 123, 12113-12114.

28 Y. Liang and R. Anwander, Microporous Mesoporous Mater., 2004, 72, 153-165.

29 F. Zeng, J. Wang, Y. Wu, Y. Yu, W. Tang, M. Yin and C. Liu, Colloids Surf., A, 2014, 441, 737-743.

30 Y. Zhang, W. W. Dai, Y. J. Liu and B. G. Ma, $R S C A d v ., 2017,7$, 8250-8257.

31 T. Cai, M. Zhou, D. Ren, G. Han and S. Guan, J. Power Sources, 2013, 231, 197-202.

32 P. Deng, Y. Shi, Y. Liu, Y. Liu and Q. Wang, Appl. Surf. Sci., 2018, 427, 894-904.

33 S. D. Gardner, C. S. K. Singamsetty, G. L. Booth, G. R. He and C. U. Pittman, Carbon, 1995, 33, 587-595.

34 T. Kwon, H. Nishihara, H. Itoi, Q. H. Yang and T. Kyotani, Langmuir, 2009, 25, 11961-11968.

35 J. S. Beck, J. C. Vartuli, W. J. Roth, M. E. Leonowicz, C. T. Kresge, K. D. Schmitt, C. T. W. Chu, D. H. Olson, E. W. Sheppard and S. B. McCullen, J. Am. Chem. Soc., 1992, 114, 10834-10843.

36 C. J. Brinker, Y. Lu, A. Sellinger and H. Fan, Adv. Mater., 1999, 11, 579-585.

37 H. Kunieda, A. Kazuyo Ozawa and K. L. Huang, J. Phys. Chem. $B, 1998,102,831-838$.

38 A. B. Fuertes, G. Lota, T. A. Centeno and E. Frackowiak, Electrochim. Acta, 2005, 50, 2799-2805.

39 X. Zhao, A. Wang, J. Yan, G. Sun, L. Sun and T. Zhang, Chem. Mater., 2010, 22, 5463-5473.

40 X. Zhao, Q. Zhang, B. Zhang, C.-M. Chen, J. Xu, A. Wang, D. S. Su and T. Zhang, RSC Adv., 2013, 3, 3578.

41 H. K. Jin, B. P. Sang, N. Ayrilmis, S. W. Oh and N. H. Kim, Composites, Part B, 2013, 46, 102-107. 